\title{
Algorithm for validation the results of computing device components' selecting
}

\author{
Marina Bolsunovskaya ${ }^{1}$, Aleksey Gintciak ${ }^{1}$, Svetlana Shirokova, ${ }^{1, *}$, and Aleksandra \\ Loginova $^{1}$ \\ ${ }^{1}$ Peter the Great St. Petersburg Polytechnic University, St. Petersburg, Russia
}

\begin{abstract}
The paper describes an algorithm for controlling the results of computer-aided design using an ontological model of computing device components (CDC) in the process of synthesizing topologies. This is implemented taking into account actual manufacturing technologies. The algorithm is intended for evaluating the variants of CDC implementation obtained in the process of computer-aided design. The developed algorithm for controlling the results of computer-aided design of computing devices' components is based on the method of organizing complex experiments and applying an ontological approach to the design of complex technical systems. The article describes the input and output data of the algorithm, the steps of the algorithm, and mathematical methods and tools used in the implementation of the algorithm. In addition, the article describes the assumptions and restrictions that are set during the operation of the algorithm.
\end{abstract}

\section{Introduction}

When developing complex technical systems, it is necessary to carry out timely and reliable control of the process and results of automated design. Moreover, topologies of the ontological model of components of computing devices are used in the synthesis process. It is necessary to take into account the actual manufacturing technology. To control the design results, an algorithm is developed using mathematical methods and models $[1,2]$.

The algorithm for controlling the results of computer-aided design of components of computing devices (hereinafter referred to as the Algorithm) is an integral part of the software package for computer-aided design of components of computing devices. The software package is designed to implement an algorithm for automated structural and parametric synthesis of electronic components of computing devices according to a set of requirements based on a genetic algorithm, an algorithm for diagnostics of projected components of computing devices, and an algorithm for monitoring the results of computeraided design of components of computing devices. This is necessary to increase the variability, manageability, and reliability of computing device components.

The process of evaluating variants of the obtained results of computer-aided design is performed for all CDC. It is performed taking into account the operating modes defined for

\footnotetext{
* Corresponding author: swchirokov@mail.ru
} 
the CDC, using a set of criteria for evaluating the CDC (for example, the cost criterion, the criterion of relative importance, the criterion of interconnectedness, etc.) [3].

The developed algorithm is designed to evaluate options for both one CDC at each time and for the DC as a whole. The authors have shown that the algorithm is able to evaluate the significance of options for implementing CDC using methods of organizing complex examinations based on the "PATTERN" method and the method of pairwise comparisons (the Analytic Hierarchy Process (Method Saati)) [4].

\section{Formalized descriptions of the algorithms mathematical base}

Mathematical model for evaluating variants of computing devices components using methods of organizing complex expert examinations.

It is proposed to use the "PATTERN" method for evaluating variants of computing device components $[5,6]$.

On the basis of the ontological model [1], the criteria for evaluating options for CDC are determined (table 1):

- $K=\left\{k_{1}, \ldots, k_{n}\right\}=\left\{k_{i} \mid i \in \overline{1, n}\right\}$ - the criteria used to evaluate options of CDCs;

- $Q=\left\{q_{1}, \ldots, q_{n}\right\}=\left\{q_{i} \mid i \in \overline{1, n}\right\}$ - the weights of the criteria;

- $C=\left\{c_{1}, \ldots, c_{m}\right\}=\left\{c_{j} \mid j \in \overline{1, m}\right\}$ - CBC options;

- $s_{i j}$ - evaluation of the $j$-th version of the CDC according to the $i$-th criterion;

- $r_{j}$ - the resulting assessment of the $j$-th variant of the CDC.

- When drawing up the matrix of correspondences, it is important that the condition for normalizing the weight coefficients of criteria (1) and estimates of CDC for each of the criteria (2) is met

$$
\begin{aligned}
\sum_{i=1}^{n} q_{i} & =1 \\
\sum_{j=1}^{m} s_{i j}=1, i & =\overline{1, n}
\end{aligned}
$$

- The resulting estimates are calculated using the formula:

$$
r_{j}=\sum_{i=1}^{n} q_{i} s_{i j}, j=\overline{1, m}, i=\overline{1, n} .
$$

- You should check that the normalization condition for the resulting estimates is met.

To do this, you must:

$$
\sum_{j=1}^{m} r_{j}=1
$$

The obtained estimates allow us to determine which of the CDC variants meets the required list of criteria to a greater extent. Sijl scores for each of the criteria can be calculated using different methods, depending on the features of the CDC, for example, scaling, ranking, pairwise comparisons, comparisons based on linguistic scales, etc [7].

When scaling, a numeric (point) scale can be set $\{0, x\}$. After that, for each option, scores are determined on $S^{r}{ }_{i j}=\{0, x\}$ a given scale for each $j$-th criterion. This value represents some " raw" estimate. The final score value is calculated as:

$$
S_{i j}=\frac{s^{r}{ }_{i j}}{\sum_{j=1}^{m} s^{r}{ }_{i j}}, \forall i \in \overline{1, n}
$$


Table 1. Matrix matching by the method of the PATTERN.

\begin{tabular}{|c|cc|c|c|c|c|c|c|}
\hline \multirow{2}{*}{ Criterion } & \multirow{2}{*}{ Criteria weights } & \multicolumn{6}{|c|}{ CDC options / Estimates of CDC options } \\
\cline { 3 - 9 } & & 1 & 2 & $\ldots$ & $j$ & $\ldots$ & $m$ \\
\hline$k_{1}$ & & $q_{1}$ & $S_{11}$ & $S_{21}$ & $\ldots$ & $S_{1 j}$ & $\ldots$ & $S_{1 m}$ \\
\hline$k_{2}$ & & $q_{2}$ & $S_{21}$ & $S_{22}$ & $\ldots$ & $S_{2 j}$ & $\ldots$ & $S_{2 m}$ \\
\hline$\ldots$ & $\ldots$ & & $\ldots$ & $\ldots$ & $\ldots$ & $\ldots$ & $\ldots$ & $\ldots$ \\
\hline$k_{i}$ & & $q_{i}$ & $S_{i 1}$ & $S_{i 2}$ & $\ldots$ & $S_{i j}$ & $\ldots$ & $S_{i m}$ \\
\hline$\ldots$ & $\ldots$ & & $\ldots$ & $\ldots$ & $\ldots$ & $\ldots$ & $\ldots$ & $\ldots$ \\
\hline$k_{n}$ & & $q_{n}$ & $S_{n 1}$ & $S_{n 2}$ & $\ldots$ & $S_{n j}$ & $\ldots$ & $S_{n m}$ \\
\hline Total: & & & $r_{1}$ & $r_{2}$ & $\ldots$ & $r_{j}$ & $\ldots$ & $r_{n}$ \\
\hline
\end{tabular}

Ranking can be used in conjunction with scaling. If we look at the scale of relations, the value $S_{i l} / S_{i j}$ measures how many times the score of the $j$-th variant by the $i$-th criterion exceeds the score of the $\mathrm{x}$-th variant, where $(j, l \in \overline{1, m})$.

Using the method of pairwise comparisons to analyze variants of components of computing devices, taking into account their evaluation criteria. The evaluation model is based on the method of pairwise comparisons (analysis of hierarchies (Method Saati)) [2,5].

In a hierarchical model structure has four levels:

$H$ (layer 0 ) - is the top level of the structure, which can be represented by an individual decision maker (DM) results of the comparison and evaluation of alternatives by the group of experts $E$ (layer 1).

$E=\left\{e_{1}, \ldots, e_{k}\right\}=\left\{e_{z} \mid z \in \overline{1, k}\right\}$ (layer 1$)$ - the level of experts, where every $z$-th expert compares pairs of criteria (layer 2) $K$ (layer 2).

$K=\left\{k_{1}, \ldots, k_{n}\right\}=\left\{k_{i} \mid i \in \overline{1, n}\right\}$ (layer 2) - the level of criteria for finding an effective (best) solution from the set of designed CDC parameters - (layer 3). - $C$ (layer 3).

$C=\left\{c_{1}, \ldots, c_{m}\right\}=\left\{c_{j} \mid j \in \overline{1, m}\right\}$ (layer 3 ) - the level of alternatives (variations in the implementation of $\mathrm{CDC}$ ), the choice of the best of which is the result of applying this model.

Then the experts (level 1) are compared in pairs by the project Manager (level 0), which allows calculating the significance estimates of each of the experts.

Next, each of the experts (level 1) compares the criteria in pairs (level 2), which allows you to calculate estimates of the significance of the criteria $[8,9]$.

At level 3, the significance estimates for each of the alternatives are calculated in the same way.

Thus, this model allows you to take into account the estimates obtained at the higher level, at all lower levels of the hierarchy. In other words, the assessment of alternatives $c_{j}, j \in \overline{1, m}$ will take into account not only the opinion of individual experts, but also the project Manager.

To calculate the evaluation of level elements, a matrix of paired comparisons is created. In order for the results obtained when applying the model to be adequate, it is necessary that the data consistency is ensured in the matrices $A$ of paired comparisons, that is, it is necessary that the matrix is consistent.

A matrix $A$ is called consistent if:

$$
a_{i j}=a_{i s} a_{s j}, i, j, s \in \overline{1, k}
$$

To ensure consistency, the following is accepted: $a_{i j}=1 / a_{j i}$.

If, when comparing elements and filling in the matrix $A$, the $s$-th row (elements $a_{s j}$ ) is known, then an arbitrary element $a_{i j}$ is defined as 


$$
a_{i j}=\frac{a_{s j}}{a_{s i}}, i, j, s \in \overline{1, k}
$$

The diagonal elements of the matrix $A$ are equal to 1 , which means that the element is evaluated relative to itself

$$
a_{i i}=1 \forall i \in \overline{1, k}
$$

Then, for this model, if there are $k$ elements at the expert level that is initially subject to evaluation, the matrix of paired dimension $k \times k$ comparisons will have the form

$$
A=\begin{gathered}
e_{1} \\
e_{1} \\
\ldots \\
\ldots \\
e_{k}
\end{gathered}\left(\begin{array}{cccc}
1 & \ldots & \ldots & e_{k} \\
\ldots & 1 & \ldots & a_{1 k} \\
\ldots & \ldots & 1 & \ldots \\
a_{k 1} & \ldots & \ldots & 1
\end{array}\right)
$$

where the matrix elements, depending on the qualitative expert assessment, can take values from 1 to 9 on the Saati scale (table 2).

Table 2. Saati Scale.

\begin{tabular}{|c|l|l|}
\hline $\begin{array}{c}\text { Quantitative } \\
\text { assessment }\end{array}$ & Qualitative assessment & Explanation \\
\hline 1 & Equal importance & Alternatives have the same rank \\
\hline 3 & Moderate superiority & $\begin{array}{l}\text { Considerations of the superiority of one alter- } \\
\text { native over the other are inconclusive }\end{array}$ \\
\hline 5 & Strong superiority & $\begin{array}{l}\text { There Is strong evidence that one alternative is } \\
\text { significantly superior }\end{array}$ \\
\hline 7 & Significant superiority & $\begin{array}{l}\text { One of the alternatives is given such a strong } \\
\text { superiority that it becomes almost significant }\end{array}$ \\
\hline 9 & Very strong superiority & $\begin{array}{l}\text { The Evidence of the superiority of one alterna- } \\
\text { tive over the other is confirmed most strongly }\end{array}$ \\
\hline $2,4,6,8$ & Intermediate values & Are Applied in the compromise case \\
\hline
\end{tabular}

After the expert performs a qualitative assessment, that is, a matrix of paired comparisons $A$ is calculated, the eigenvector of this matrix is calculated, called the vector of priorities (expert preferences) or the vector of weight coefficients. Elements of this vector will indicate the assessment of the significance of each expert [10].

In order to calculate the vector of weight coefficients, a normalized matrix $A$ of the form must be created for the matrix

$$
N_{A}=\left(\begin{array}{cccc}
\frac{a_{11}}{a_{11}+\ldots+a_{k 1}} & \ldots & \ldots & \frac{a_{1 k}}{a_{1 k}+\ldots+a_{k k}} \\
\ldots & \ldots & \ldots & \ldots \\
\ldots & \ldots & \ldots & \ldots \\
\frac{a_{k 1}}{a_{11}+\ldots+a_{k 1}} & \ldots & \ldots & \frac{a_{k k}}{a_{1 k}+\ldots+a_{k k}}
\end{array}\right),
$$

after that, you can proceed to calculating the estimates as the average values of the elements of the corresponding rows of the matrix $N_{A}$. Then the priority vector for level 1 of the hierarchy will look like 


$$
W_{k \times 1}^{1}=\left(\begin{array}{c}
w_{e_{1}} \\
\ldots \\
w_{e_{k}}
\end{array}\right)_{k \times 1}=\left(\begin{array}{c}
\left(\frac{a_{11}}{a_{11}+\ldots+a_{k 1}}+\ldots+\frac{a_{1 k}}{a_{1 k}+\ldots+a_{k k}}\right) / k \\
\ldots a_{k 1} \\
\left(\frac{a_{11}+\ldots+a_{k 1}}{a_{1}}+\ldots+\frac{a_{k k}}{a_{1 k}+\ldots+a_{k k}}\right) / k
\end{array}\right)_{k \times 1},
$$

where the normalization condition must be met $w_{e_{1}}+\ldots+w_{e_{k}}=1$.

Thus, the values of the vector $W^{1}$ at the first hierarchical level represent estimates of relative importance, which are attributed to the point of view of each of the experts regarding the selection process.

After describing the calculations for the first level of the hierarchical structure, you need to make the following remark. All calculated vectors of weight coefficients are eigenvectors of the corresponding matrices. You can also find the values of these vectors by solving the equation $A W=\lambda W$, where $\lambda$-is the proper value of the matrix. The condition of consistency of the matrix is formed as $A W=n W$.

Then the values found are more accurate the closer $\lambda_{\max }$ to $n$, i.e. the matrix of paired comparisons reflects the agreed opinions of experts only when $\lambda_{\max }=>\mathrm{n}$.

Deviation $\lambda_{\max }$ from $\mathrm{n}$ can serve as a measure of consistency of expert judgments. The consistency index is used $C I$ as an indicator of the consistency measure):

$$
C I=\frac{\lambda_{\max }}{n-1}
$$

The consistency ratio $\mathrm{CR}$ (consistency ratio) is used to evaluate the degree of consistency):

$$
C R=\frac{C I}{C I S}
$$

where $C I S$ is determined experimentally as the average value of the coefficient $C I$ for a large sample of randomly generated matrices of paired comparisons. Table 3 shows the values $C I S$ for matrices of paired comparisons of different dimensions.

The judgments are consistent if the value $C R$ does not exceed 0.1 (in some cases, for large-dimensional matrices, 0.2). Exceeding the set value indicates the need to revise judgments about the elements of the matrix of paired comparisons [7].

Table 3. Values $C I S$ for paired comparison matrices.

\begin{tabular}{|l|l|l|l|l|l|l|l|l|l|l|}
\hline $\mathrm{n}$ & 3 & 4 & 5 & 6 & 7 & 8 & 9 & 10 & 11 & 12 \\
\hline CIS & 0.58 & 0.9 & 1.12 & 1.24 & 1.32 & 1.41 & 1.45 & 1.49 & 1.51 & 1.48 \\
\hline
\end{tabular}

At the second level of the hierarchical structure, each $i$-th criterion is compared by each $z$-th expert. Then for each of the experts $e_{1}, \ldots, e_{k}$, in accordance with the number $n$ of criteria specified at the second level of the model, a matrix $n \times n$ of paired comparisons will be created

$$
A_{e 1}=\begin{array}{cccc}
k_{1} & \ldots & \ldots & k_{n} \\
k_{1} \\
\ldots \\
k_{n}
\end{array}\left(\begin{array}{cccc}
1 & \ldots & \ldots & a_{1 n} \\
\ldots & 1 & \ldots & \ldots \\
\ldots & \ldots & 1 & \ldots \\
a_{n 1} & \ldots & \ldots & 1
\end{array}\right), \ldots, A_{e k}=\begin{array}{r}
k_{1} \\
k_{1} \\
\ldots \\
k_{n}
\end{array}\left(\begin{array}{cccc}
1 & \ldots & \ldots & k_{n} \\
\ldots & 1 & \ldots & \ldots \\
\ldots & \ldots & 1 & \ldots \\
a_{n 1} & \ldots & \ldots & 1
\end{array}\right) .
$$

Then, similarly to the 1-st level of the structure, normalized matrices $N_{A_{e 1}}, \ldots, N_{A_{e k}}$ and, accordingly, vectors of weight coefficients $W^{A_{e 1}}, \ldots, W^{A_{e k}}$ are calculated. Then to get a vector for the second level, it is necessary to form a matrix $\Psi_{n \times k}$ from $k$ the obtained vectors $W^{A_{e 1}}, \ldots, W^{A_{e k}}$, each of which will have a size $n \times 1$. Then the expression 


$$
W_{n \times 1}^{2}=\Psi_{n \times k}^{e} \times W_{k \times 1}^{1}=\left(\begin{array}{c}
w_{k_{1}} \\
\cdots \\
w_{k_{n}}
\end{array}\right)_{n \times 1}
$$

allows you to determine the vector of weight coefficients for the 2 nd level of the hierarchy, and the normalization condition $w_{k_{1}}+\ldots+w_{k_{n}}=1$ must be met. Thus, the weight coefficients of the vector $W^{2}$ mean an assessment of the relative importance of each of the criteria, taking into account the opinion of the expert group.

Alternatives (level 3 objects) are compared by each $z$-th expert according to each $i$-th criterion. Then, according to the number of alternatives $(m)$, a pair comparison matrix $(n)$ will be created $A_{k 1}, \ldots, A_{k n}$, each of which has a size $m \times m[8]$.

Similarly to the previous levels, normalized matrices $N_{A_{k 1}}, \ldots, N_{A_{k n}}$ and, accordingly, vectors of weight coefficients $W^{A_{k 1}}, \ldots, W^{A_{k n}}$ are calculated. Then to get a vector for the third level, it is necessary to form a matrix $\Psi^{k}{ }_{m \times n}$ from the $(n)$ obtained vectors $W^{A_{k 1}}, \ldots, W^{A_{k n}}$, each of which will have a size $m \times 1$. Then the expression will allow you to define the vector of weight coefficients for the 3rd level of the hierarchy

$$
W^{3}{ }_{m \times 1}=\Psi^{e}{ }_{m \times n} \times W^{2}{ }_{n \times 1}=\left(\begin{array}{c}
w_{c_{1}} \\
\cdots \\
w_{c_{m}}
\end{array}\right)_{m \times 1},
$$

where $W^{2}{ }_{n \times 1}$ - is calculated according to (15), and the normalization condition $w_{c_{1}}+\ldots+w_{c_{m}}=1$ must also be met here. The resulting vector of weight coefficients $W^{3}$ determines the assessment of the significance of each alternative.

If we consider each branch of the hierarchy separately, it turns out that at the third level of the hierarchy for each $z$-th expert $(z \in \overline{1, k})$, we will get $n$ matrices of paired comparisons in accordance with the number of criteria by which the expert evaluates alternatives.

Matrices will be constructed $A_{1}{ }^{k_{1}}, \ldots, A_{1}{ }^{k_{n}}, \ldots, A_{z}{ }^{k_{1}}, \ldots, A_{z}{ }^{k_{n}}, \ldots, A_{k}{ }^{k_{1}}, \ldots, A_{k}{ }^{k_{n}}$, each of which will have a dimension $m \times m$ in accordance with the number of alternatives. Accordingly, normalized $N_{A_{1}} k_{1}, \ldots, N_{A_{1}} k_{n}, \ldots, N_{A_{Z}} k_{1}, \ldots, N_{A_{Z}} k_{n}, \ldots, N_{A_{k}} k_{1}, \ldots, N_{A_{k} k_{n}}$ and priority vectors $W^{A_{1}{ }^{k_{1}}}, \ldots, W^{A_{1}{ }^{k_{n}}}, \ldots, W^{A_{k}{ }^{k_{1}}}, \ldots, W^{A_{k}{ }^{k_{n}}}$ of dimension $m \times 1$ will be calculated. Then for each of the $z$ experts it is necessary to calculate the value of the vector

$$
W^{A_{z}{ }^{K}}=\left(\begin{array}{c}
w^{Z}{ }_{c_{1}} \\
\cdots \\
w^{Z}{ }_{c_{m}}
\end{array}\right)_{m \times 1}=\psi_{m \times n}^{z} \times W^{2}{ }_{n \times 1},
$$

where $z \in \overline{1, k}, \psi^{z}{ }_{m \times n}$ - a matrix consisting of vectors $W^{A_{z}{ }^{k_{1}}}, \ldots, W^{A_{z}{ }^{k_{n}}}, K$ - set of all criteria, $W^{2}{ }_{n \times 1}$-vector of weight coefficients of the 2 nd level, calculated by the formula (17). The vector of weight coefficients of the 3 rd level will be calculated as

$$
W_{m \times 1}^{3}=\left(\begin{array}{c}
w_{c_{1}} \\
\cdots \\
w_{c_{m}}
\end{array}\right)_{m \times 1}=\psi_{m \times k} \times W_{k \times 1}^{1},
$$

where $z \in \overline{1, k}, \psi_{m \times k^{-}}$a matrix consisting of vectors $W^{A_{1}{ }^{K}}, \ldots, W^{A_{z}{ }^{K}} \ldots, W^{A_{k}{ }^{K}}$, calculated by formula (17), $W^{1}{ }_{k \times 1}$ is a vector of weight coefficients of the 1 st level calculated by formula (11). 


\section{Results}

The software package provides the following functions:

a) configuration of computing devices based on actual manufacturing technologies;

b) diagnosis of the components of computing devices;

c) detection of anomalies (failures) in the operation of the designed computer device components;

d) the notification of the occurrence of abnormal situations;

e) formation of recommendations for the prevention of abnormal situations;

f) analysis of variants of components of computing devices obtained in the course of computer-aided design;

g) calculating estimates of the relative importance of alternatives to the designed components of computing devices [11,12].

The input data of the software package is:

a) the specified configuration of the $\mathrm{CD}$ (computing device) in the configuration file format;

b) input data of algorithms for design and diagnostics of components of computing devices;

c) statistical data on the functioning of the $\mathrm{CD}$;

d) criteria for evaluating variants of components of computing devices obtained during computer-aided design.

The algorithm is used to evaluate the options designed by the CDC in different configurations.

The authors have developed algorithms for evaluating options for CDC and monitoring the design results [13].

Algorithm for evaluating variants of computing device components using the "PATTERN" method.

Step 1. Determine all the options for the CDC in the CD structure for evaluation.

Step 2. For each CDC to construct a tree topology, where.

Step3. For each CDC variant, perform Step 2 recursively, traversing the CDC topology tree in the direction of the lowest-level CDC (elementary component).

Step 4. Select the evaluation criterion for the CDC variant, which will be used to calculate the CDC variant score.

Step 5. Based on the selected criterion, taking into account the tree of the CDC topology, determine the estimates of the CDC variant.

Step 6. Repeat steps 4 and 5 for all the criteria for the selected CDC option.

Step 7. For each option, where Steps 2 to 6 to execute recursively and create a table of matching variants of CDCs.

Step 8. Check the condition for normalizing the weight coefficients of the criteria (1) and the CDC estimates for each of the criteria (2). If the normalization condition is not met, go back to step 7.

Step 9. To carry out calculation of the resulting estimated version of CDCs.

Step 10. Check that the normalization condition for the resulting estimates (4) is met. If the normalization condition is not met, go back to step 9 .

Step 11. Rank in descending order of the resulting ratings options CDC.

Algorithm for using the method of pairwise comparisons for analyzing the parameters of computing devices components, taking into account their evaluation criteria.

Step 1. Determine all the options for the CDC in the CD structure for evaluation.

Step 2. The project Manager (level 0) should create a list of experts (level 1) and perform their pairwise comparison on the Saati scale (table 2), form a normalized matrix in accordance with the calculation formula (10), and a priority vector in accordance with the 
calculation formula (11), which will allow calculating the significance ratings of each of the experts.

Step 3. Check the matrix of paired expert comparisons for consistency using the formula (6). If the consistency condition is not met, go back to step 2.

Step 4. Each of the experts (level 1) should compare the criteria (level 2) in pairs on the Saati scale (table 2), form a matrix in accordance with the calculation formula (14), and the priority vector in accordance with the calculation formula (15), which will allow calculating the significance of the criteria.

Step 5. Check the matrix of paired comparisons of criteria for consistency using the formula (6). If the consistency condition is not met, go back to step 4.

Step 6. At level 3, similarly calculate the significance estimates of each CDC variant on the Saati scale (table 2), form a matrix in accordance with the calculation formula (16), and a priority vector in accordance with the calculation formula (17).

Step 7. Check the matrix of paired comparisons of CDC variants for consistency using the formula (6). If the consistency condition is not met, go back to step 6 .

Step 8. Calculate the resulting estimates of the CDC options, taking into account the assessment of the significance of each of the experts.

Step 9. Rank the CDC variants in descending order of the resulting ratings [13].

Algorithm for controlling the results of computer-aided design using an ontological model of computing device components in the process of topology synthesis, taking into account real manufacturing technologies.

The algorithm assumes the formation of an integral assessment of the CDC options, taking into account the criteria for their assessment on the basis of:

1) an Algorithm for evaluating options for CDC using the "PATTERN" method»;

2) an Algorithm for using the pairwise comparison method (the Saati hierarchy analysis method) to analyze the variants of CDC.

Step 1. Determine all the options for the CDC in the CD structure for evaluation.

Step 2. Generate the resulting estimates of CDC variants using the algorithm for evaluating CDC variants using the "PATTERN" method.

Step 3. Generate the resulting estimates of the CDC variants using the pairwise comparison method (Saati hierarchy analysis method).

Step 4. Create a vector of priorities for the algorithms for evaluating HQ options using the "PATTERN" method and the method of pairwise comparisons (the method of analyzing Saati hierarchies), which will allow calculating the significance estimates of the resulting estimates of HQ options obtained after performing the algorhythms.

Step 5. Perform calculation of integral estimates of HQ variants taking into account the priority vector of algorithms for evaluating HQ variants formed at Step 4.

Step 6. Rank in descending order of integral estimates the options of the CDC [14].

\section{Conclusion}

The result of solving the algorithm for controlling the results of computer-aided design of components of computing devices is a structured tabular representation of the resulting estimates. These are estimates of options for implementing the CDC in the form of a set of estimates of CDC options for each criterion. Assessments are considered within the framework of a single method of organizing complex examinations and, if necessary, in General, in each specific moment of time. The input data for the algorithm is information about the CDC variants $[15,16]$. Under the integral assessment of the implementation of CDCs using the selected method of complex expertise is a set of estimates of the embodiment of CDC according to separate criteria. The list of criteria, their number and order of formation is determined by the chosen method of organizing complex 
examinations. The input data for the algorithm is information about the criteria for evaluating options for implementing components of computing devices, and the procedure for calculating the resulting estimates for each criterion within each of the methods used.

The input data of the control algorithm must be synchronized within the framework of an ontological model describing the functioning of components of computing devices. They are input to the algorithm as a structure for each component. The control algorithm returns an estimate of the implementation options for individual components at a given time in the form of estimates for a given set of criteria. The list of criteria is determined by the requirements for the $\mathrm{CDC}$ and the chosen method of organizing complex examinations [17].

Output data is presented for each CDC as a whole in the form of a structured table description.

The research is partially funded by the Ministry of Science and Higher Education of the Russian Federation as part of World-class Research Center program: Advanced Digital Technologies (contract No. 075-15-2020-934).

\section{References}

1. O.G. Kantor, S.I. Spivak, Informatics and its application 8, 2, 111-121 (2014)

2. V.N. Volkova, G.V. Gorelova, A.A. Efremov, et al, Systems and processes modeling (Moscow: Yurait Publ., 2016)

3. V.N. Volkova, Theory of information systems. Textbook (St. Petersburg: SaintPetersburg Polytechnic University Publ., 2014)

4. V.N. Volkova, Yu.Yu. Cherny, The laws of information processes in open systems. Rethinking L. Von Bertalanffy Proc. Int. Conf. SARC (2016)

5. V.N. Volkova, V.N. Kozlov, V.E. Mager, L.V. Chernenkaya, Classification of methods and models in system analysis. Proc. 20th IEEE International Conference on Soft Computing and Measurements SCM-2017 (St. Petersburg: ETU “LETI”)

6. M.V. Bolsunovskaya, S.V. Shirokova, A.V. Loginova, M.B. Uspenskiy, Development of tools for improving the data storage systems reliability as a part of digital transformation strategy, IOP Conference Series: Materials Science and Engineering 940(1), 012010 (2020)

7. V.N. Volkova, Systems theory and systems analysis in the management of organizations (2013)

8. A.A. Denisov, Introduction to information systems analysis (Leningrad: LPI, 1980)

9. A.A. Denisov, Modern problems of system analysis (2008)

10. M. Bolsunovskaya, S. Shirokova, A. Loginova, State of current data storage market and development of tools for increasing data storage systems reliability, E3S Web of Conferences 135, 04076 (2019)

11. V. Venkatesan, Reliability Analysis of Data Storage Systems: These pour l'obtention du grade de docteur es sciences. (Lausanne: École polytechnique fédérale de Lausanne, 2012)

12. V. Venkatesan, I. liadis, A general reliability model for data storage systems Proc. 9th International Conference on Quantitative Evaluation of Systems 209-219 (2012)

13. T. Yang, et al, DEBAR: A scalable high-performance de-duplication storage system for backup and archiving. Proc. IEEE International Symposium on Parallel \& 


$\begin{array}{llllll}\text { Distributed } & \text { Processing } & \text { (IPDPS) } & 1-12 & \text { (IEEE) }\end{array}$

\section{DOI:10.1109/IPDPS.2010.5470468}

14. W. Avani, et al, Protecting against rare event failures in archival systems Proc. IEEE International Symposium on Modeling, Analysis \& Simulation of Computer and Telecommunication Systems (London UK) 1-11 (IEEE) (2009) DOI: 10.1109/MASCOT.2009.5366825

15. Q. Xin, T.J.E. Schwarz, E.L. Miller, Disk infant mortality in large storage systems Proc. 13th IEEE International Symposium on Modeling, Analysis, and Simulation of Computer and Telecommunication Systems 125-134 (IEEE) (2005)

16. J.J. Wylie, et al, Selecting the right data distribution scheme for a survivable storage system. School of Computer Science (Pittsburgh: Carnegie Mellon University, 2001)

17. A.S. Makarov, M.V. Bolsunovskaya, S.V. Shirokova, M.B. Uspenskij, A.A. Kuz'michjov, Proc. Int. Conf. SCM-2018 (St. Petersburg: ETU “LETI”, 2018) 